\title{
Nitrate decreases ruminal methane production with slight changes to ruminal methanogen composition of nitrate- adapted steers
}

\author{
Liping Zhao, Qingxiang Meng, Yan Li, Hao Wu, Yunlong Huo, Xinzhuang Zhang and Zhenming Zhou* (D)
}

\begin{abstract}
Background: This study was conducted to examine effects of nitrate on ruminal methane production, methanogen abundance, and composition. Six rumen-fistulated LimousinxJinnan steers were fed diets supplemented with either $0 \%(0 N R), 1 \%(1 N R)$, or 2\% (2NR) nitrate (dry matter basis) regimens in succession. Rumen fluid was taken after two-week adaptation for evaluation of in vitro methane production, methanogen abundance, and composition measurements.
\end{abstract}

Results: Results showed that nitrate significantly decreased in vitro ruminal methane production at $6 \mathrm{~h}, 12 \mathrm{~h}$, and $24 \mathrm{~h}$ $(P<0.01 ; P<0.01 ; P=0.01)$. The $1 \mathrm{NR}$ and $2 \mathrm{NR}$ regimens numerically reduced the methanogen population by $4.47 \%$ and $25.82 \%$ respectively. However, there was no significant difference observed between treatments. The alpha and beta diversity of the methanogen community was not significantly changed by nitrate either. However, the relative abundance of the methanogen genera was greatly changed. Methanosphaera $\left(P_{L}=0.0033\right)$ and Methanimicrococcus $\left(P_{L}=0.0113\right)$ abundance increased linearly commensurate with increasing nitration levels, while Methanoplanus abundance was significantly decreased $\left(P_{L}=0.0013\right)$. The population of Methanoculleus, the least frequently identified genus in this study, exhibited quadratic growth from $0 \%$ to $2 \%$ when nitrate was added $\left(P_{Q}=0.0140\right)$.

Conclusions: Correlation analysis found that methane reduction was significantly related to Methanobrevibacter and Methanoplanus abundance, and negatively correlated with Methanosphaera and Methanimicrococcus abundance.

Keywords: Nitrate, Methane, Methanogen diversity, Hiseq sequencing

\section{Background}

Globally, around $40 \%$ of anthropogenic methane from the agricultural sector is produced by livestock [1] and methane production results in approximately a $2-5 \%$ energy loss from feed [2]. Several additives have been tested to lower methane production from ruminants such as saponin, sulfate, and nitrate $[3,4]$. To reduce methane production effectively, it is essential to have a good understanding of the population and distribution of ruminal methanogens. Yanagita et al. [5] reported that $2.8 \%$ to $4.0 \%$ of microorganisms in the rumen have the

\footnotetext{
* Correspondence: zhouzm@cau.edu.cn

State Key Laboratory of Animal Nutrition, College of Animal Science and Technology, China Agricultural University, Beijing 100193, People's Republic of China
}

(c) The Author(s). 2018 Open Access This article is distributed under the terms of the Creative Commons Attribution 4.0 International License (http://creativecommons.org/licenses/by/4.0/), which permits unrestricted use, distribution, and reproduction in any medium, provided you give appropriate credit to the original author(s) and the source, provide a link to the Creative Commons license, and indicate if changes were made. The Creative Commons Public Domain Dedication waiver (http://creativecommons.org/publicdomain/zero/1.0/) applies to the data made available in this article, unless otherwise stated. tured because of strictly anaerobic characteristics. Studies relating to the $16 \mathrm{~s}$ rRNA gene of the archaea and methyl-coenzyme $M$ reductase gene $(m c r A)$ found that the abundant methanogenic groups in the rumen are Methanobrevibacter, Methanomicrobium and Rumen cluster C (RCC) [4, 6-8].

As a potential ruminal methane inhibitor, nitrate could change the rumen bacterial community composition in steers (Lin et al., 2013) in the following two ways: 1) toxicity by nitrite, an intermediate of nitrate reduction; 2) competition for hydrogen. Even though methane production is undesirable, methanogens play an irreplaceable role in maintaining rumen function by consuming hydrogen produced through rumen fermentation. Studies have 
suggested that nitrate- and nitrite-reducing microbes compete with methanogen for hydrogen in the rumen, making nitrate an alternative hydrogen sink that mitigates against ruminal methane production $[9,10]$. The theory behind this is that nitrate reduction is energetically more beneficial than methanogenesis in thermodynamics [11]. This is because the electrochemical reduction of $1 \mathrm{~mol} \mathrm{ni-}$ trate to ammonia consumes $8 \mathrm{~mol}$ of electrons resulting in hydrogen formation [12]. Researchers believe that nitrate could greatly decrease ruminal methanogen population in unadapted ruminants $[13,14]$. However, in practice, it is controversial whether nitrate can persistently inhibit methane production and methanogen populations in nitrate-adapted ruminants. Shi et al. [15] reported that the suppression effect disappeared after adaptation to nitrate, while van Zijderveld et al. found that nitrate could effectively and persistently suppress ruminal methane production in dairy cows [13]. These contrasting results could be due to the different methanogen diversities and structures in wither and dairy cattle [4]. However, limited studies have been conducted to assess the methanogenic communities in nitrate-adapted beef cattle.

This study was conducted to investigate methane production from Limousin-crossed steers fed with or without nitrate using an in vitro gas production technique. Methanogen abundance and diversity was also examined using real-time quantitative PCR and high-resolution technology Hiseq sequencing to understand the changes caused by nitrate addition and to ascertain the reasons why methane production could be inhibited by nitrate.

\section{Methods}

\section{Animals}

The study treatments and rations have previously been described in a report by Zhao et al. [16]. Briefly, six Limousin $\times$ Jinnan crossbreed steers $(450 \pm 20 \mathrm{~kg})$ with permanent rumen fistula, located at Jiweifuren feedlot, were fed with mixed rations consisting of $30 \%$ concentrate and 70\% corn straw. In addition, three levels of nitrate were added (on a dry matter basis): $0 \%$ (0NR), $1 \%$ (1NR), and $2 \%$ (2NR). Urea was added to 0NR and $1 \mathrm{NR}$ regimens to maintain the iso-nitrogen level among treatments (Table 1). Two-week adaptation was adopted for steers and ruminal microbes before sampling, and nitrate addition through $1 \mathrm{NR}$ and $2 \mathrm{NR}$ treatments was

Table 1 Chemical composition of diets

\begin{tabular}{llll}
\hline Items & \multicolumn{3}{l}{ Treatments } \\
\cline { 2 - 4 } & ONR & 1NR & 2NR \\
\hline Metabolizable energy (MJ/kg) & 7.30 & 7.30 & 7.30 \\
Crude protein (\%DM) & 11.41 & 11.41 & 11.41 \\
Ca (\%DM) & 0.64 & 0.64 & 0.64 \\
P (\%DM) & 0.24 & 0.24 & 0.24 \\
\hline
\end{tabular}

gradually increased to the target level during the adaptation period in order to avoid nitrite accumulation in the rumen. After adaptation, rumen fluid was taken before the morning feed for in vitro gas production and $6 \mathrm{~h}$ post-feed for DNA extraction and methanogenic community diversity measurements. For testing of methanogen community diversity, rumen fluid was frozen immediately in liquid nitrogen after sampling and then transferred to $-80^{\circ} \mathrm{C}$.

\section{In vitro gas production technique}

Rumen fluid taken before the morning feed on the fifteenth day was stored in a $39^{\circ} \mathrm{C}$ pre-warmed syringe thermos, then carried immediately back to the laboratory immediately for modified in vitro gas production according to the procedure of Menke et al. [17]. The detailed procedure was previously described in Zhao et al. [18] (more information in Additional file 1). Gas from $6 \mathrm{~h}, 12 \mathrm{~h}$, and $24 \mathrm{~h}$ incubation was sampled for methane production measurement using gas chromatography (TP-2060 T, Beijing Analytical Instrument Co., Ltd, China) equipped with a TCD detector (column: TDX-01, $1 \mathrm{~m} \times 3 \mathrm{~mm} \times 2 \mathrm{~mm}$ ).

\section{DNA extraction and methanogen quantification}

Total genomic DNA from 17 samples was extracted while one 2NR sample was excluded due to improper storage. A DNA extraction toolkit was adopted for DNA extraction (Tiangen Biotech Co., Beijing, China) combined with an oscillator (Precellys 24, Bertin Technology, Montigny-le-Bretonneux, France). Rotating speed of the oscillator was $5500 \mathrm{rpm}$ with two circulations and $30 \mathrm{~s}$ per circulation.

Total methanogen abundance was quantified with an ABI 7300 Prism real-time PCR (ABI, Foster City, CA, USA) using SYBR Green PCR RealMaster Mix (Tiangen Biotech, co., LTD, China). Methanogen 16 s rRNA sequences were amplified using primers Met630F/Met803R (Met630F-GGATTAGATACCCSGGTAGT; Met803R-RG TTGARTCCAATTAAACCGCA) [19] on the following PCR program: one cycle of $95{ }^{\circ} \mathrm{C}$ for $15 \mathrm{~min}$ (initial denaturation), 35 cycles of $95{ }^{\circ} \mathrm{C}$ for $30 \mathrm{~s}$ (denaturation), $60{ }^{\circ} \mathrm{C}$ for $30 \mathrm{~s}$ (annealing) and $72{ }^{\circ} \mathrm{C}$ for $30 \mathrm{~s}$ (elongation), followed by a final step of $72{ }^{\circ} \mathrm{C}$ for $5 \mathrm{~min}$. All real-time PCR assays were performed in triplicate.

\section{Hiseq sequencing and data analyses}

The archaeal 16S rRNA genomic sequence was amplified with the primers Arc344F/Arc519R (Arc344F-ACGGG GYGCAGCAGGCGCGA; Arc519R-GWATTACCGCG GCKGCTG) [20]. Amplicon purification was performed using a Qiagen MinElute PCR purification kit (Qiagen, Valencia, CA, USA) and the quantity of amplicon libraries was estimated with the Qubitds DNA HS assay using 
a Qubit 2.0 instrument (Life Technologies, USA). Subsequently, Illumina paired-end sequencing libraries were constructed from the purified PCR products using a NEB Next ${ }^{\circ}$ Ultra $^{\text {Tx }}$ DNA Library Prep Kit for Illumina (NEB, USA). Quality control of the amplicon libraries was performed using BioRad Experion. Finally, the libraries were sequenced on a Illumina HiSeq 2500 platform (Illumina Inc., San Diego, USA), which was used to produce paired-end sequence reads with cyclic reversible chain termination chemistry.

Sequenced paired-end reads were merged with FLASH and then grouped according to the attached barcode with QIIME software [21]. Quality control was applied using the NGS QC Toolkit [22] with the following two criteria: 1) keeping sequences with a score of 32 consecutive bases all greater than 27 ; 2) removing chimeras. All samples were subsampled to equal size of 300,000 reads, because an even depth of sampling is required for beta diversity calculations. The $16 \mathrm{~S}$ rRNA operational taxonomic units (OTUs) were defined at the 3\% dissimilarity threshold with uclust (v1.2.22). A total of 9736 OTUs were obtained for all samples and the average OTU quantity for each treatment was 2.071 for 0NR; 1946 for 1NR; and 1984 for 2NR. The number of sequences and OTUs is presented in Additional file 2: Table S1. The rarefaction curve (Additional file 3: Figure S1) and alpha diversity indices (ACE, Chao1, Shannon, and Simpson) were developed on the basis of OTUs. Furthermore, a phylogenetic tree for beta diversity metrics was generated with FastTree [23] based on the aligned sequences, and visualization was confirmed using principal coordinates analyses for the weighted UniFrac distances. The rarefaction curve indicated that a reasonable number of individual samples had been taken. The representative sequence of each OTU, which was the most abundant sequence, was assigned to the lowest possible taxonomic rank with RDP Classifier [24], and a reference dataset from the Greengene database was used. A Venn diagram of OTUs for different treatments was plotted. Correlation coefficients between methane production and methanogenic genus were calculated using multivariate analysis of variance in SAS and results were plotted with corrplot $\mathrm{R}$ software [25].

\section{Statistical analysis}

Gas composition, methanogen abundance, methanogenic community composition, and diversity indices were analyzed with a general linear effects model using SAS 9.0 (SAS Institute, Cary, NC, USA). Furthermore, linear and quadratic tendencies were analyzed. Statistical significance was set to $P<0.05$. Differences between treatments were assessed with Duncan's new multiple range test.

\section{Results}

\section{Methane production and methanogen abundance}

Figure 1 showed the changes in methane proportions among steers adapted to the three nitrate diets. Nitrate dramatically decreased the in vitro methane proportion of gas production at $6 \mathrm{~h}(P<0.01), 12 \mathrm{~h}(\mathrm{P}<0.01)$ and $24 \mathrm{~h}(P=0.01)$ fermentation. Ruminal methanogen population of Limousin crossed steers fed with urea was $2.66 \times 106$ copies $/ \mu \mathrm{l}$ (Fig. 2). Nitrate linearly decreased methanogen abundance by $4.47 \%$ for $1 \mathrm{NR}$ and $25.82 \%$ for $2 \mathrm{NR}$ compared to 0NR. However, no significantly statistical difference was detected between the three treatments $(P=0.53)$.

\section{General information of sequencing results}

In total, 9736 OTUs were obtained in this study for all samples at the $3 \%$ dissimilarity threshold. The number

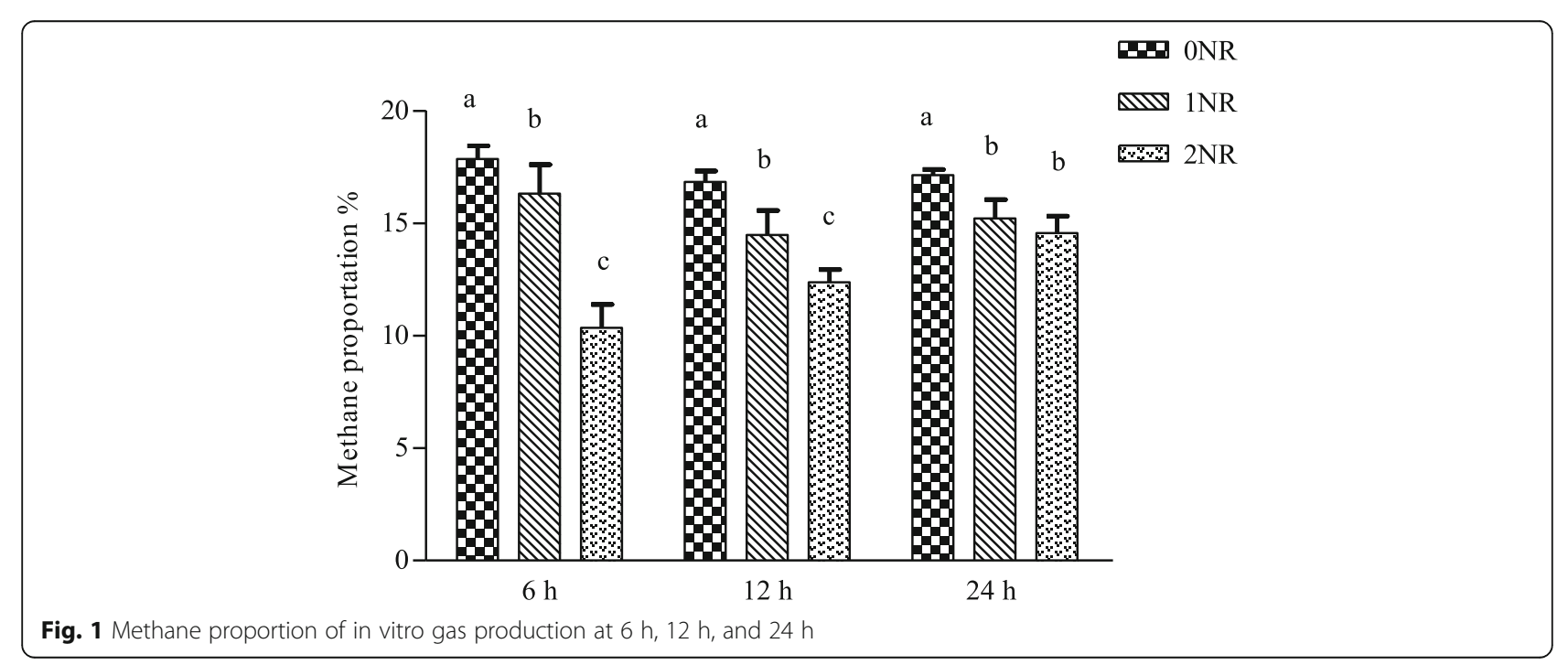




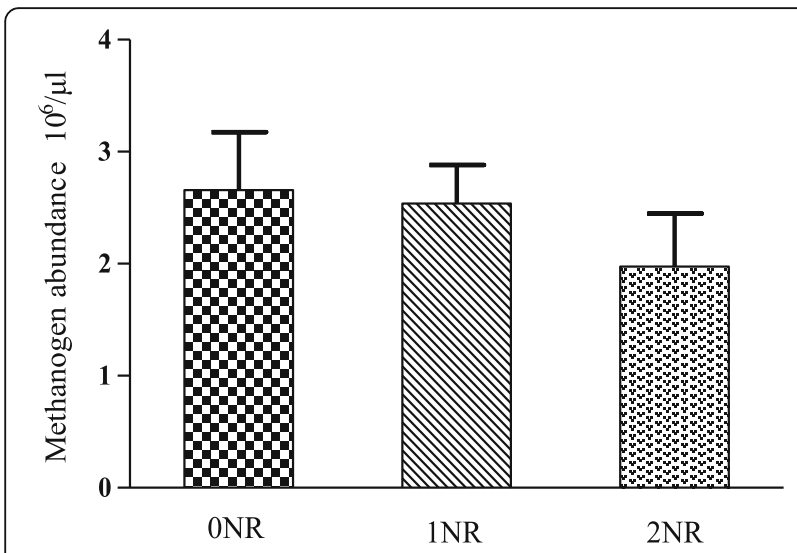

Fig. 2 Changes in ruminal methanogen abundance by treatments

of OTUs exclusively found in $0 \mathrm{NR}, 1 \mathrm{NR}$ and $2 \mathrm{NR}$ treatments were 1446 (14.85\%), 1254 (12.88\%) and 1042 (10.70\%) respectively, while shared OTUs between two treatments were 1462 (15.02\%) for 0NR and 1NR, 1128 (11.59\%) for 0NR and 2NR, and 1044 (10.72\%) for $1 \mathrm{NR}$ and 2NR separately. Furthermore, there were 2360 OTUs (24.24\%) shared for the three treatments (Fig. 3). The coverage of sequencing was between 99.53-99.59\%, which meant that the sequencing was detailed enough to cover all 16 s rRNA sequences (Additional file 2: Table S1). The average ACE and Chao1 index of all samples was 6352 and 5789, and the average Simpson and Shannon scores were 0.86 and 3.85 , respectively.
Methanobacteria, Methanomicrobia, and RCC Thermoplasmata were classified in this study as the three methanogenic classes (Fig. 4). RCC Thermoplasmata and Methanobacteria were the two main classes with high abundance, accounting for $56.11 \%$ and $37.32 \%$ of total sequences, while Methanomicrobia exhibited considerably lower abundance accounting for $0.54 \%$. In addition, approximately $6.02 \%$ sequences could not be assigned to any archaea class. Furthermore, 4 orders, 4 families and 7 genera of methanogenic groups were detected when sequences were further analyzed at a more detailed level (not shown).

At the genus level, the Methanobrevibacter from Methanobacteria and vadinCA11 from RCC Thermoplasmata were the main genera found in the samples, accounting for $35.82 \%$ and $56.09 \%$ of total sequences, respectively. In addition to the highly abundant genera, some other less prevalent genera were detected accounting for $1.25 \%$ of sequences in total. These included Methanosphaera (1.02\%), Methanimicrococcus $(0.22 \%)$, Methanosarcina (0.01\%), Methanobacterium (0.003\%), Methanoplanus (0.002\%) and Methanoculleus (0.0005\%).

\section{Effects of nitrate on methanogen composition}

Statistical analysis of the relative abundance of methanogenic orders are presented in Table 2. The majority of methanogenic orders E2 and Methanobacteriales were not significantly affected by nitrate addition $(P=0.51$; $P=0.42)$. However, Methanomicrobiales $(P<0.05)$ and

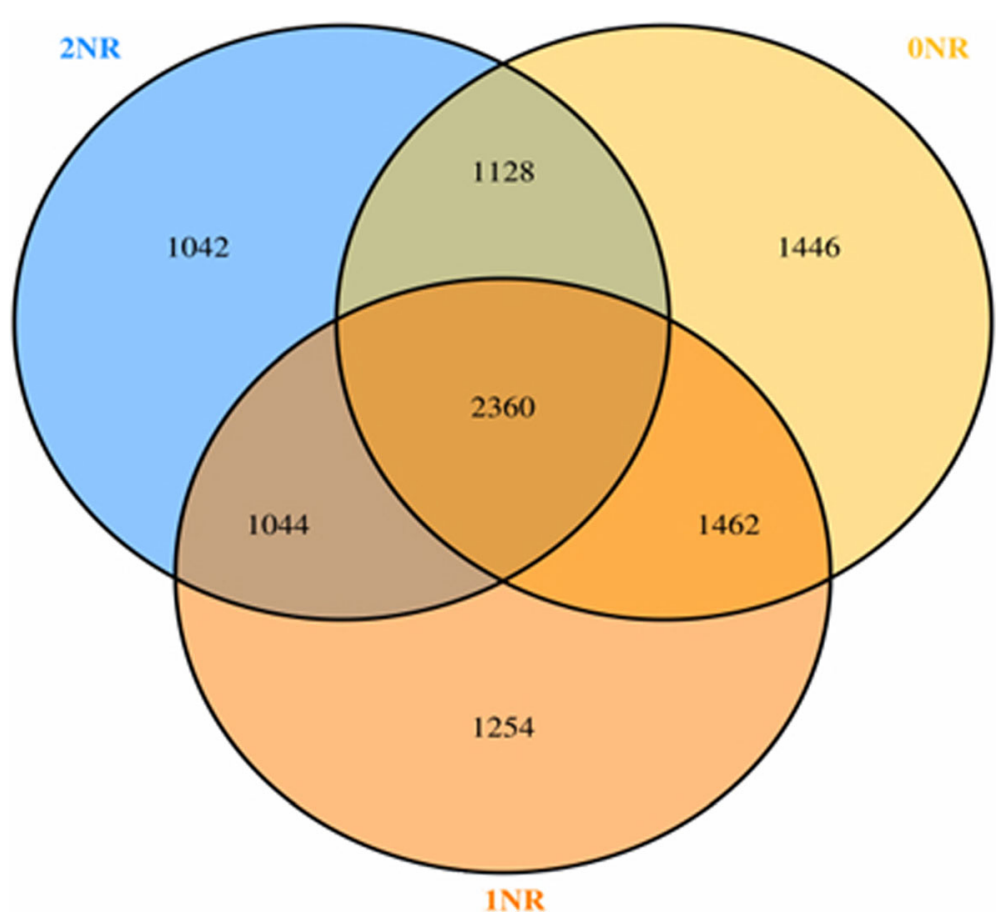

Fig. 3 Venn diagram for overlap between observed OTUs at 3\% dissimilarity of ONR, 1NR and 2NR 


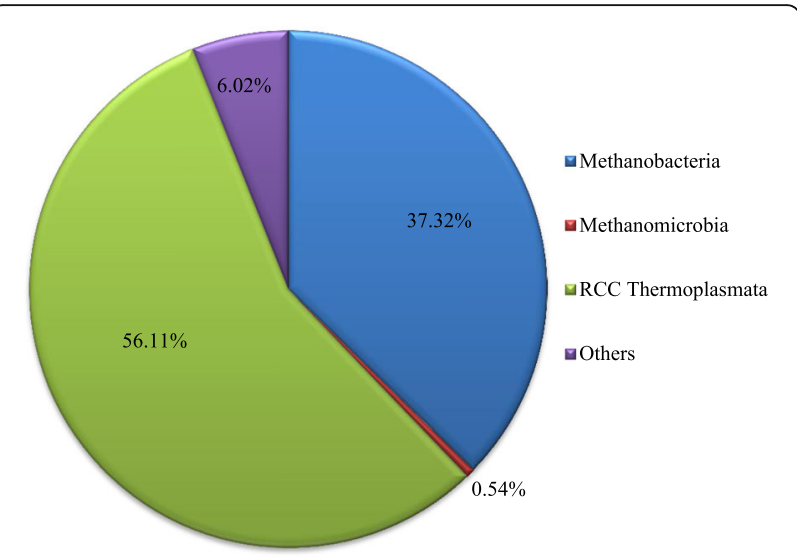

Fig. 4 Average relative abundance of methanogenic class in the rumen of steers

Methanosarcinales $(P=0.01)$ were significantly influenced by nitrate addition, whose abundance were decreased on a linear basis and increased commensurate with increased nitrate addition $(P<0.05)$.

Changes in methanogen genera abundance caused by nitrate are listed in Table 3. Similar to the changes in orders, the relative abundance of the majority of the genera in this study, vadinCA11 and Methanobrevibacter, were not significantly influenced by nitration addition $(\mathrm{P}=0.51 ; P=0.49)$. However, the abundance of the least prevalent genera were significantly affected with increased nitrate addition. Methanosphaera $\left(\mathrm{P}_{\mathrm{L}}=0.0033\right)$ and Methanimicrococcus $\left(\mathrm{P}_{\mathrm{L}}=0.0113\right)$ abundance increased linearly commensurate with augmented nitration addition resulting in a significantly higher abundance for $2 \mathrm{NR}$. With respect to low prevalence genera, growth of Methanoplanus was significantly suppressed by nitrate $(P=0.0028)$. The abundance of Methanoculleus, the smallest genus detected in this study, showed quadratic changes from $0 \mathrm{NR}$ to $2 \mathrm{NR}\left(\mathrm{P}_{\mathrm{Q}}=0.0140\right)$.

Based on sequencing results, nitrate did not significantly affect alpha or beta diversity of ruminal methanogen (Table 4 and Additional file 4: Figure S2). The alpha diversity indices (ACE, Chao1, Shannon, and Simpson) of the ruminal archaeal populations were not significantly affected by nitrate

Table 2 Effects of nitrate on relative abundance of methanogen order of rumen

\begin{tabular}{|c|c|c|c|c|c|c|c|}
\hline \multirow[t]{2}{*}{ Items } & \multicolumn{3}{|c|}{ Treatments } & \multirow[t]{2}{*}{ SEM } & \multirow[t]{2}{*}{$P$-value } & \multicolumn{2}{|c|}{ Contrast } \\
\hline & ONR & $1 \mathrm{NR}$ & $2 N R$ & & & $\overline{P_{L}}$ & $P_{Q}$ \\
\hline $\bar{E} 2$ & 56.15 & 54.15 & 58.34 & 2.62 & 0.51 & 0.55 & 0.32 \\
\hline Methanobacteriales & 38.00 & 35.68 & 34.62 & 2.12 & 0.42 & 0.20 & 0.80 \\
\hline Methanomicrobiales & $0.47^{\mathrm{a}}$ & $0.25^{b}$ & $0.27^{b}$ & 0.06 & $<0.05$ & 0.05 & 0.13 \\
\hline Methanosarcinales & $0.06^{\mathrm{b}}$ & $0.12^{\mathrm{b}}$ & $0.24^{\mathrm{a}}$ & 0.03 & 0.01 & $<0.01$ & 0.40 \\
\hline
\end{tabular}

supplementation $\quad(P=0.89 ; \quad P=0.66 ; \quad P=0.18 ; \quad P=0.05$; Table 4). The comparisons between methanogen communities by PCoA (Additional file 4: Figure S2) based on weighted UniFrac distance revealed no difference among treatments, indicating that nitrate addition did not impact upon the ruminal archaeal population.

\section{Relationships between methane production and methanogen genera}

Correlations between methane production and abundance of the methanogen genera were presented in Fig. 5. Methanobrevibacter from Methanobacterials and Methanoplanus from Methanomicrobials were positively related with in vitro methane production at $6 \mathrm{~h}, 12 \mathrm{~h}$, and $24 \mathrm{~h}$. Methanobrevibacter had a strongly positive relationship with methane production at $6 \mathrm{~h}$ and $12 \mathrm{~h}$ $\left(R^{2}=0.89 ; R^{2}=0.59\right)$, while Methanoplanus abundance had a persistently positive correlation with methane production $\left(R^{2}=0.73 ; R^{2}=0.97 ; R^{2}=0.99\right)$. Methanosphaera from Methanobacterials $\left(R^{2}=0.98 ; R^{2}=0.77 ; R^{2}=0.54\right)$ and Methanimicrococcus from Methanomicrobials $\left(R^{2}=0.95 ; R^{2}=0.98 ; R^{2}=0.86\right)$ were negatively correlated with in vitro methane production at $6 \mathrm{~h}, 12 \mathrm{~h}$, and $24 \mathrm{~h}$. VadinCA11 from the E2 order was negatively correlated with methane production at $6 \mathrm{~h}\left(\mathrm{R}^{2}=0.59\right)$.

\section{Discussion}

Manipulating ruminal methane production is a research focus of several disciplines between ruminant nutrition and environmental science. In the rumen, methanogenesis evolves from carbon oxide, acetic acids, and methanol by methanogens. For most methanogens, methanogensis from $\mathrm{CO}_{2}$ and $\mathrm{H}_{2}$ is the sole energy source. The process of methanogensis is affected by many environmental factors, such as carbohydrate type, digestion passage rate, and feed additives, as well as internal factors. These include: 1) the inhibition of methanogen growth and abundance, 2) the reduction of hydrogen donors and or hydrogen competition from other electron acceptors and 3) gene expression and activity inhibition of critical enzymes $[3,26,27]$. The mechanisms to decrease ruminal methane production vary but provision of hydrogen acceptors to compete with electrons as a methane precursor is an effective method [28]. Nitrate has a higher electron competing ability compared with carbon dioxide making it an effective enteric methane production inhibitor $[13,29]$. Aside from the toxicity of nitrite, the intermediate of nitrate reduction in the rumen, it is also considered effective in methane inhibition. Consistent with previous results of van Zijderveld et al. [30], we found that nitrate could persistently reduce methane production of steers after a two-week adaptation period in this study. However, Shi et al. [11] observed conflicting results where inhibition disappeared when sheep were adapted to nitrate rations. 
Table 3 Effects of nitrate on relative abundance of methanogenic genera

\begin{tabular}{|c|c|c|c|c|c|c|c|}
\hline \multirow[t]{2}{*}{ Item } & \multicolumn{3}{|c|}{ Treatments } & \multirow[t]{2}{*}{ SEM } & \multirow[t]{2}{*}{ P-value } & \multicolumn{2}{|c|}{ Contrast } \\
\hline & ONR & $1 \mathrm{NR}$ & $2 N R$ & & & $P_{L}$ & $P_{Q}$ \\
\hline vadinCA11 & 56.15 & 54.15 & 58.34 & 2.62 & 0.51 & 0.55 & 0.32 \\
\hline Methanobrevibacter & 36.64 & 35.43 & 32.89 & 2.20 & 0.49 & 0.25 & 0.81 \\
\hline Methanosphaera & $0.8100^{b}$ & $0.8550^{\mathrm{b}}$ & $1.3575^{\mathrm{a}}$ & 0.0977 & 0.0057 & 0.0033 & 0.0881 \\
\hline Methanimicrococcus & $0.1000^{b}$ & $0.1017^{b}$ & $0.2300^{\mathrm{a}}$ & 0.0333 & 0.0199 & 0.0113 & 0.0979 \\
\hline Methanoplanus & $0.0033^{\mathrm{a}}$ & $0.0012^{b}$ & $0.0009^{b}$ & 0.0005 & 0.0028 & 0.0013 & 0.1508 \\
\hline Methanosarcina & 0.0029 & 0.0045 & 0.0028 & 0.0007 & 0.2141 & 0.9550 & 0.0855 \\
\hline Methanobacterium & 0.0019 & 0.0014 & 0.0013 & 0.0004 & 0.4066 & 0.2816 & 0.4590 \\
\hline Methanoculleus & $0.0003^{b}$ & $0.0010^{\mathrm{a}}$ & $0.0003^{b}$ & 0.0002 & 0.0417 & 1.0000 & 0.0140 \\
\hline
\end{tabular}

ONR control, $1 N R 1 \%$ nitrate, $2 N R 2 \%$ nitrate, $\mathrm{P}_{\mathrm{L}}$ is liner tendency; $\mathrm{P}_{\mathrm{Q}}$ is quadratic tendency

These conflicting results might result from the different animal types, diet base, and environments where these studies were performed.

As previously described, methanogenesis generated from $\mathrm{CO}_{2}$ and $\mathrm{H}_{2}$ is the sole energy source for most methanogens. Therefore, consistently adding methane inhibitors to ruminants will mitigate methane production and result in inhibited growth of methanogens [4]. Nitrate can effectively suppress methane production because its reduction is a more favourable pathway for the hydrogen sink than the reduction of carbon dioxide to methane [13], resulting in decreased ruminal methanogen abundance. Furthermore, the extent of decline is more obvious with increased levels of nitrate that are added [14]. Therefore, methanogen abundance numerically decreased when nitrate was introduced to nitrateadapted steers but not significantly, which means methane reduction is not due to total population shifts between ruminants fed with and without nitrate.

Methanogens are taxonomically categorised as Methanobacteria, Methanococci, Methanomicrobia, Methanopyri and RCC [32]. However, few classes have been consistently found at high abundance because of the different animal species, diets, environments, and DNA extraction methods [31-34]. More detailed assessment of rumen fluid samples using Hiseq sequencing technology provided a more intricate view of the archaea than

Table 4 Changes in bacterial richness and biodiversity by nitrate

\begin{tabular}{|c|c|c|c|c|c|c|c|}
\hline \multirow[t]{2}{*}{ Item } & \multicolumn{3}{|c|}{ Treatments } & \multirow[t]{2}{*}{ SEM } & \multirow[t]{2}{*}{$P$-value } & \multicolumn{2}{|c|}{ Contrast } \\
\hline & $\overline{O N R}$ & $1 \mathrm{NR}$ & $2 N R$ & & & $\overline{P_{L}}$ & $P_{Q}$ \\
\hline$\overline{\mathrm{ACE}}$ & 6375.5 & 6280.1 & 6316.5 & 138.19 & 0.89 & 0.78 & 0.71 \\
\hline Chaol & 5643.7 & 5513.9 & 5684.1 & 145.64 & 0.66 & 0.84 & 0.38 \\
\hline Shannon & 0.86 & 0.85 & 0.87 & 0.01 & 0.18 & 0.36 & 0.10 \\
\hline Simpson & 3.86 & 3.75 & 3.95 & 0.05 & 0.05 & 0.23 & 0.03 \\
\hline
\end{tabular}

DDGE and TGGE methods [34]. RCC Thermoplasmata, Methanobacteria and Methanomicrobia were the three main archaeal classes in Limousin crossed steers, accounting for $99.46 \%$ of all methanogens. At the genus level, vadinCA11 from Thermoplasmata and Methanobrevibacter from Methanobacteria were the top two abundant genera in the rumen, which is different from other studies [6, 34]. Consistent with the results of Pei et al., nitrate addition did not greatly change the alpha and beta diversity of ruminal methanogens [7]. However, the taxonomic community was slightly changed by nitrate addition.

We postulate that the mechanism of nitrate influencing methanogen abundance could be hydrogen competition and nitrite toxicity to methanogens [14]. Nitrate is more competitive for hydrogen than $\mathrm{CO}_{2}$ and methanol [13], which could decrease energy provided for methanogen metabolism and growth. As previously mentioned, most methanogens attain their energy from $\mathrm{CO}_{2}$ and methanol reduction, which means that most methanogens are bacteria, which require hydrogen as a source of nutrition. These include Methanobrevibacter, Methanosphaera, Methanoplanus, Methanobacterium, and Methanimicrococcus [6]. Nitrate decreased the abundance of Methanoplanus, Methanobacterium, and Methanobrevibacter significantly or numerically due to hydrogen competition. The numerical decline of Methanobacterium and Methanobrevibacter results from high variation between duplicates within treatments. VadinCA11 from RCC Thermoplasmata, the most abundant genus in this study, does not have a representative species within culture [35]. Its energy resource is methylamine but this has not been proven to be hydrogenotrophic [36]. The reason for the increases in Methanimicrococcus and Methanosphaera abundance associated with $2 \mathrm{NR}$ is unknown.

As previously mentioned, the changes in methanogen structure caused by nitrate might be a reason for methane inhibition [37, 38]. Correlation analysis found that methane reduction caused by nitrate addition was 


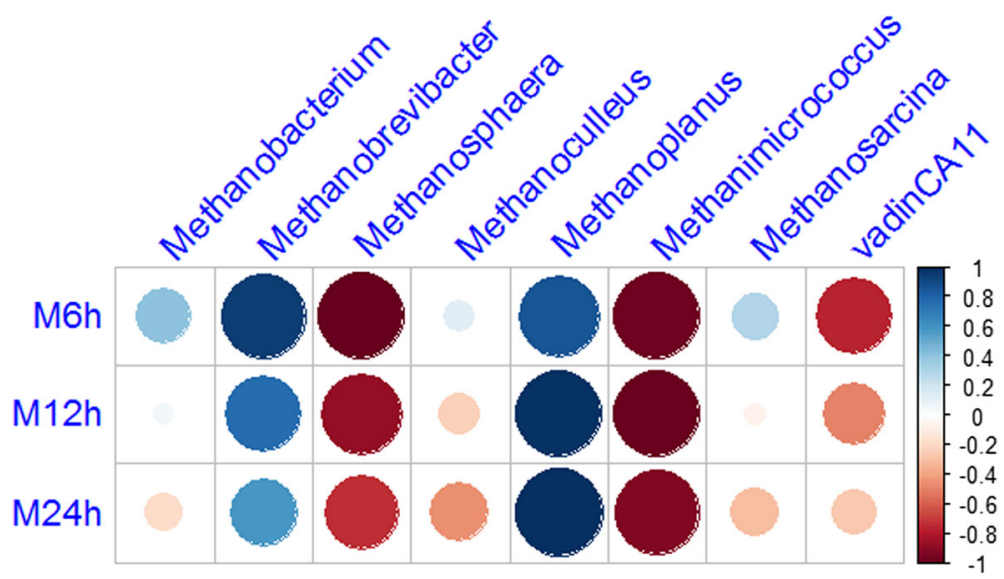

Fig. 5 Correlation between methane production and methanogen genera abundance

positively related to the reduction of Methanobrevibacter and Methanoplanus abundance and negatively correlated with an increase in the Methanosphaera, Methanimicrococcus, and VadinCA11. However, the abundances of Methanobrevibacter and VadinCA11 were numerically changed depending on treatments so the changes may not be stable. Methanoplanus, Methanosphaera, and Methanimicrococcus are found at low abundance in the rumen i.e. less than $1 \%$. Therefore, the resultant shifts in methanogen community structure in response to nitrate treatment appear to have minor effects on methane reduction. There must be other reasons accounting for methane inhibition in the rumen by nitrate. For example, the inhibition of expression of methyl coenzyme $M$ reductase gene and the reduction of enzyme activity could be one of the reasons. Further studies need to be performed to determine these hypotheses and an accurate correlation needs to be drawn between methyl coenzyme $M$ reductase activity and in vivo gas production in future studies.

\section{Conclusion}

Nitrate additive greatly reduced methane production of nitrate-adapted steers. However, its influence on methanogen abundance and diversity was minor, as it only changed the abundance of less prevalent genera, such as Methanoplanus (negatively) and Methanimicrococcus and Methanosphaera (both positively). Correlation analysis found that there was a strong positive relationship with Methanobrevibacter and Methanoplanus abundance, and methane reduction. However, methane reduction was negatively correlated with Methanosphaera and Methanimicrococcus abundance. However, their contribution to methane reduction was not influential because of the insignificance of Methanobrevibacter between treatments and the low abundance of Methanoplanus, Methanosphaera and Methanimicrococcus.
Therefore, further studies are required to consider other factors and their contribution to methane mitigation. An example could be gene expression and activity of key enzymes of methanogenesis (methyl coenzyme $M$ reductase) in nitrate-adapted animals.

\section{Additional file}

Additional file 1: In vitro gas production technique. (PDF $140 \mathrm{~kb}$ ) Additional file 2: Table S1. Reads and OTUs abundance of 165 rRNA gene libraries. (PDF $99 \mathrm{~kb}$ )

Additional file 3: Figure S1. Rarefaction curve for each sample with 97\% similarity as threshold. (PNG $149 \mathrm{~kb}$ )

Additional file 4: Figure S2. Principal coordinates analysis showing relationships of methanogen abundance among treatments. (PNG $31 \mathrm{~kb}$ )

\section{Abbreviations}

ACE: Abundance-based coverage estimator; DGGE: Denaturing gradient gel electrophoresis; DM: Dry matter; OTUs: Operational taxonomic units;

PCoA: Principal coordinate analysis; PCR: Polymerase chain reaction; SEM: Standard error of mean

\section{Acknowledgments}

We thank Xiaodong Li and Jinwei Zhao for their technical assistance. The authors also wish to acknowledge the students at the Beef Cattle Research Center of CAU for their support on this experiment. We are grateful to the native English speaking scientists of Elixigen Company for editing our manuscript.

\section{Funding}

This study was supported by the National Natural Science Fund of China (No. 31672449, 31101738), the National Scientific Program (2011CB302705), and the China Agricultural Research System (CARS-37). The funding bodies had no role in the design of the study or collection, analysis or interpretation of data or in writing the manuscript.

\section{Availability of data and materials}

The data supporting the conclusion of this article are included within the article and 16S rRNA gene sequences supporting the results of this article are available in the GenBank Database (https://www.ncbi.nlm.nih.gov/ genbank/) under accession numbers SRR4299450.

Authors' contributions

LPZ, QXM, YLH, XZZ and ZMZ designed the study. LPZ, YL, HW, YLH and XZZ participated in the animal experiment and biochemical and molecular 
experiments, LPZ analyzed the data and drafted the manuscript. LPZ, QXM, $Y L H, X Z Z$ and ZMZ revised the manuscript. All authors read and approved the final manuscript.

\section{Competing interest}

The authors declare that they have no competing interests.

\section{Ethics approval}

The protocol was approved by the China Agricultural University Animal Welfare and Ethical Committee (Permit No. DK1406). Experiments were performed in accordance with the Regulations for the Administration of Affairs Concerning Experimental Animals (The State Science and Technology Commission of P. R. China, 1988).

\section{Consent for publication}

Not applicable.

\section{Publisher's Note}

Springer Nature remains neutral with regard to jurisdictional claims in published maps and institutional affiliations.

\section{Received: 11 May 2017 Accepted: 15 March 2018}

Published online: 20 March 2018

\section{References}

1. Key N, Tallard G. Mitigating methane emissions from livestock: a globa analysis of sectoral policies. Clim Chang. 2012;112(2):387-414.

2. Johnson KA, Johnson DE. Methane emissions from cattle. J Anim Sci. 1995; 73(8):2483-92

3. Benchaar C, Pomar C, Chiquette J. Evaluation of dietary strategies to reduce methane production in ruminants: a modelling approach. Can J Anim Sci. 2001;81(4):563-74.

4. Patra AK, Yu Z. Combinations of nitrate, saponin, and sulfate additively reduce methane production by rumen cultures in vitro while not adversely affecting feed digestion, fermentation or microbial communities. Bioresour Technol. 2014;155:129-35.

5. Yanagita K, Kamagata Y, Kawaharasaki M, et al. Phylogenetic analysis of methanogens in sheep rumen ecosystem and detection of Methanomicrobium mobile by fluorescence in situ hybridization. Biosci Biotechnol Biochem. 2000;64(8):1737-42.

6. Janssen PH, Kirs M. Structure of the archaeal community of the rumen. Appl Environ Microbiol. 2008:74(12):3619-25.

7. Pei CX, Mao SY, Cheng YF, et al. Diversity, abundance and novel 165 rRNA gene sequences of methanogens in rumen liquid, solid and epithelium fractions of Jinnan cattle. Animal. 2010;4(01):20-9.

8. Kittelmann $\mathrm{S}$, Seedorf $\mathrm{H}$, Walters WA, et al. Simultaneous amplicon sequencing to explore co-occurrence patterns of bacterial, archaeal and eukaryotic microorganisms in rumen microbial communities. PLoS One. 2013:8(2):e47879.

9. Hulshof RBA, Berndt A, Gerrits WJJ, et al. Dietary nitrate supplementation reduces methane emission in beef cattle fed sugarcane-based diets. J Anim Sci. 2012;90(7):2317-23.

10. Leng RA. Interactions between microbial consortia in biofilms: a paradigm shift in rumen microbial ecology and enteric methane mitigation. Anim Prod Sci. 2014;54(5):519-43.

11. Guo WS, Schaefer DM, Guo XX, et al. Use of nitrate-nitrogen as a sole dietary nitrogen source to inhibit ruminal methanogenesis and to improve microbial nitrogen synthesis in vitro. Asian Australas J Anim Sci. 2009:22(4):542-9.

12. Richardson DJ, Watmough NJ. Inorganic nitrogen metabolism in bacteria. Curr Opin Chem Biol. 1999:3:207-19.

13. Zhou Z, Yu Z, Meng Q. Effects of nitrate on methane production, fermentation, and microbial populations in in vitro ruminal cultures. Bioresour Technol. 2012:103(1):173-9.

14. Van Zijderveld SM, Gerrits WJ, Dijkstra J, et al. Persistency of methane mitigation by dietary nitrate supplementation in dairy cows. J Dairy Sci. 2011;94(8):4028-38.

15. Shi C, Meng Q, Hou X, et al. Response of ruminal fermentation, methane production and dry matter digestibility to microbial source and nitrate addition level in an in vitro incubation with rumen microbes obtained from wethers. J Anim Vet Adv. 2012;11:3334-41.
16. Li L, Davis J, Nolan J, et al. An initial investigation on rumen fermentation pattern and methane emission of sheep offered diets containing urea or nitrate as the nitrogen source. Anim Prod Sci. 2012;52(7):653-8.

17. Zhao L, Ren L, Zhou Z, et al. Improving ruminal degradability and energetic values of bamboo shoot shell using chemical treatments. Anim Sci J. 87:896-903.

18. Zhao L, Meng $Q$, Ren $L$, et al. Effects of nitrate addition on rumen fermentation, bacterial biodiversity and abundance. Asian-Australas J Anim Sci. 2015;28(10):1433-41.

19. Menke KH, Raab L, Salewski A, et al. The estimation of the digestibility and metabolizable energy content of ruminant feedingstuffs from the gas production when they are incubated with rumen liquor in vitro. J Agric Sci. 1979:93(01):217-22

20. Hook SE, Northwood KS, Wright ADG, et al. Long-term monensin supplementation does not significantly affect the quantity or diversity of methanogens in the rumen of the lactating dairy cow. Appl Environ Microbiol. 2009;75(2):374-80.

21. Yu Z, García-González R, Schanbacher FL, et al. Evaluations of different hypervariable regions of archaeal 165 rRNA genes in profiling of methanogens by archaea-specific PCR and denaturing gradient gel electrophoresis. Appl Environ Microbiol. 2008;74(3):889-93.

22. Langmead B, Salzberg SL. Fast gapped-read alignment with bowtie 2. Nat Methods. 2012;9(4):357-9.

23. Caporaso JG, Kuczynski J, Stombaugh J, et al. QIIME allows analysis of highthroughput community sequencing data. Nat Methods. 2010;7(5):335-6.

24. Patel R K, Jain M. NGS QC Toolkit: a toolkit for quality control of next generation sequencing data. PLoS One, 2012, 7(2): e30619.

25. Price MN, Dehal PS, Arkin AP. FastTree: computing large minimum evolution trees with profiles instead of a distance matrix. Mol Biol Evol. 2009;26(7):1641-50

26. DeSantis TZ, Hugenholtz P, Larsen N, et al. Greengenes, a chimera-checked $16 \mathrm{~S}$ rRNA gene database and workbench compatible with ARB. Appl Environ Microbiol. 2006:72(7):5069-72

27. Wei T. corrplot: Visualization of a correlation matrix. R package version 0.73 , 2013, 230(231): 11

28. Hironaka R, Mathison GW, Kerrigan BK, et al. The effect of pelleting of alfalfa hay on methane production and digestibility by steers. Sci Total Environ. 1996;180(3):221-7.

29. McAllister TA, Cheng KJ, Okine EK, et al. Dietary, environmental and microbiological aspects of methane production in ruminants. Can J Anim Sci. 1996;76(2):231-43.

30. Van Zijderveld SM, Gerrits WJJ, Apajalahti JA, et al. Nitrate and sulfate: effective alternative hydrogen sinks for mitigation of ruminal methane production in sheep. J Dairy Sci. 2010;93(12):5856-66.

31. Marais JP, Therion JJ, Mackie Rl, et al. Effect of nitrate and its reduction products on the growth and activity of the rumen microbial population. $\mathrm{Br}$ J Nutr. 1988;59(02):301-13.

32. Newbold JR, Van Zijderveld SM, Hulshof RBA, et al. The effect of incremental levels of dietary nitrate on methane emissions in Holstein steers and performance in Nelore bulls. J Anim Sci. 2014:92(11):5032-40.

33. Dridi B, Fardeau ML, Ollivier B, et al. Methanomassiliicoccus luminyensis gen Nov., sp. nov., a methanogenic archaeon isolated from human faeces. Int Syst Evol Microbiol. 2012;62(8):1902-7.

34. Whitford MF, Teather RM, Forster RJ. Phylogenetic analysis of methanogens from the bovine rumen. BMC Microbiol. 2001:1(1):5.

35. Wright ADG, Williams AJ, Winder B, et al. Molecular diversity of rumen methanogens from sheep in Western Australia. Appl Environ Microbiol. 2004;70(3):1263-70.

36. Franzolin R, St-Pierre B, Northwood K, et al. Analysis of rumen methanogen diversity in water buffaloes (Bubalus bubalis) under three different diets. Microb Ecol. 2012:64(1):131-9.

37. Goodrich JK, Waters $J$, Poole AC, et al. Human genetics shape the gut microbiome. Cell. 2014;159(4):789-99.

38. Poulsen M, Schwab C, Jensen BB, et al. Methylotrophic methanogenic Thermoplasmata implicated in reduced methane emissions from bovine rumen. Nat Commun. 2013:4:1428. 